\title{
Brain MR image denoising based on wavelet transform
}

\author{
Deepti Gupta* and Musheer Ahmad \\ Department of Applied Sciences \& Humanities, Jamia Millia Islamia, New Delhi, India
}

\section{(C2018 ACCENTS}

\begin{abstract}
Images must be clear and noise free, in order to achieve better accuracy in classification results of brain tumor from magnetic resonance imaging (MRI). But in the process of collection of medical images, the picture gets noisy, inadvertently. Deletion of noise from images is known as wavelet shrinkage or thresholding. In this work, an ingenious and compatible method is proposed for the valuation of thresholding parameters, hinge on the information of wavelet coefficients. For the better illustration of the process brain MRI was introduced with Gaussian noise at the different level of variances and then denoised using Wavelet Transform with coding in MATLAB. The same procedure was repeated to denoise three brain MR Images with the brain tumor. Proposed method helps in embellished off the edge and texture details of the images. The image quality of brain MR images is assessed in terms of peak signal-to-noise ratio (PSNR). Experimental results represent that this method attain preferable denoised image with improved PSNR.
\end{abstract}

\section{Keywords}

Magnetic resonance imaging (MRI), Image denoising, Thresholding function, Peak signal to noise ratio (PSNR).

\section{Introduction}

Noise is a disturbance which occurs during acquisition and transmission of images from one medium to another. It may corrupt image and provide an erroneous repercussion during image processing. Image quality can generally be upgraded by removing noise and enhancing contrast. Preferably, at the time of removal of noise from images as many as possible significant features should be preserved. Medical images acquired from CT Scan, MRI and Xray are the standard tool for diagnosis in the medical field. These images are often exaggerated by random noise and Gaussian noise. An existence of noise generates adverse visual quality and reduces the visibility of minimal contrast objects. Noise removal is vital in medical imaging applications to get hidden details of the data and enhance image quality. Medical images are normally contaminated with noise, which obstructs the medical finding related to these images. There is a widespread concern in the problem of denoising images in traditional devices from the traditional image processing area to the MRI images have been applied for denoising, However, the practice of noise inhibition must not considerably corrupt the valuable features in an image. In general, edges are significant features for medical images and hence the denoising must be balanced with edge preservation [1].

*Author for correspondence
Wavelets are well accepted for medical image denoising and enhancement applications for their good localization properties both in space and frequency. Wavelets do not isolate the smoothness along the edges, and thus more suitable for reconstruction of sharp point singularities than lines or edges [2]. The wavelet transform is progressively applied in image denoising domain for their intrinsic worth furthermore, its functioning is easy, and its application performance is good, wavelet transform has been widely paid attention across the globe [3].

\section{Related work}

In general, the denoising methods are linear likewise Wiener filter, but one of the drawbacks of Wiener filter and Gaussian filter denoising is it consists image blur situation. In recent times, nonlinear systems are derived from wavelet transforms are more operable [4]. Elyasi et al. are the investigators of the initial denoising papers using wavelet [5]. In 2009 they have presented that the noise might be considerably diminished with persistence of edge sharpness by wavelet thresholding. Donoho and Johnstone have established various principal theoretical results namely wavelet shrinkage with higher convergence rate $[6,7]$. One of the standard methods namely, Bayes shrink in which the threshold is acquired by means of the Bayesian method [8, 9]. Bayes Shrink method is a subband related it means that the thresholding is performed at each subband in 
the wavelet decomposition. It is studied as smoothness adaptive. Further efforts made in the domain of wavelet thresholding is reviewed in [1013]. They proposed a new thresholding method using soft-thresholding. They demonstrated that their proposed method outperforms the traditional ones in terms of PSNR; thus, improving the denoised results significantly [14]. Simulation results are also given to show the efficacy of our proposed method.

Denoising by the methods of Wiener filter and Gaussian filter causes the edge blurred situation. Bilateral filtering [11] is the highest intensive nonlinear smoothing filter, in spite of the fact that it undergoes the gradient reversal effect, which employs an intricate computation based histogram approximation to figure out the weight. In recent times, Zhang et al. [9] create an augmented bilateral filter based structure which is competent of effectively get rid of the noise. It receives spatial information and grayscale similarity from images and attains denoising along with edge-preserving.

\subsection{Available threshold function for image denoising}

Let us take an original image $k_{i j}$ of size $\mathrm{NxN}$ and add the Gaussian noise $m_{i j}$ to original image to make it noisy image $n_{i j}$, i.e.,

$$
n_{i j}=k_{i j}+m_{i j}
$$

Apply the wavelet transform to (1) to obtain the wavelet coefficients $F_{i j}$. Convert the wavelet coefficients $F_{i j}$ by applying the soft thresholding [7]. and then take inverse-wavelet transform to get the denoised image $\hat{f}$.

$\hat{f}=\left\{\begin{array}{lc}\mathrm{F}_{\mathrm{ij}}-\mathrm{t}, & \text { if } \mathrm{F}_{\mathrm{ij}} \geq \mathrm{t} \\ \mathrm{F}_{\mathrm{ij}}+\mathrm{t}, & \text { if } \mathrm{F}_{\mathrm{ij}} \leq-\mathrm{t} \\ 0, & \text { if }\left|\mathrm{F}_{\mathrm{ij}}\right|<\mathrm{t}\end{array}\right.$

Where $t$ is the threshold value.

Donoho et al. [7] have discussed a simple but influential wavelet-based denoising pattern known as VisuShrink. The outcomes of VisuShrink are stable along with an alluring visual feature. Even though VisuShrink is liable to over-smooth the signal, but fail to retain details like sharp edges of the original signal that come out in the increased estimation error. VisuShrink uses the Universal threshold, S, which is proportional to the standard deviation of the noise, is defined as [6]:

12

$$
S=\sigma \sqrt{2 \log N}
$$

Where $\sigma^{2}$ is the noise variance defined as given in following equation.

$\sigma^{2}=\left[\left(\operatorname{median}\left|n_{i j}\right|\right) / 0.06745\right]^{2}$

Where $n_{i j} \in H H_{1}$ subband thresholding.

$\mathrm{N}$ : Number of a pixel for the test image.

\section{Proposed denoising method}

With this proposed technique we are obtaining denoised images afterward estimation of new thresholding function.

\subsection{Evaluation of proposed thresholding function} To get an improved value of the thresholding is our main task. An undersized threshold will outpace all the noisy coefficients [15]. So, noise remains in the denoised image. On the contrary, a wide threshold value label an extra number of coefficients as zero, which destroy some important details by converting the noisy signal into smooth and it may create blurs and artifact [16, 4]. Therefore, we try to explore optimum threshold technique. The proposed technique is adaptive to dissimilar sub-band characteristics by investigating the parameters of the wavelet coefficients as follows [17]:

$A(p)=\sum_{i, j} F_{i j}$

For $\mathrm{p}=0,1$ and 2 , the $F_{i j}$ are the wavelet coefficients for horizontal, vertical and diagonal values respectively.

$$
T=\frac{\sum_{0}^{2} A(p)}{\widehat{N}}
$$

Where $\widehat{N}=N / 2^{b}$, here $\mathrm{b}=1,2, \ldots, \mathrm{k}$ and $\mathrm{k}$ denotes the number of decompositions.

Threshold Factor

$Q=\exp \{(S-T) /(S+T)\}$

Now, we evaluate a new threshold value $S_{\text {new }}$ as given in $\mathrm{eq}^{\mathrm{n}}(8)$

$S_{\text {new }}=\sigma Q$

With this new threshold parameter, we will get the denoised image from the noisy image. 


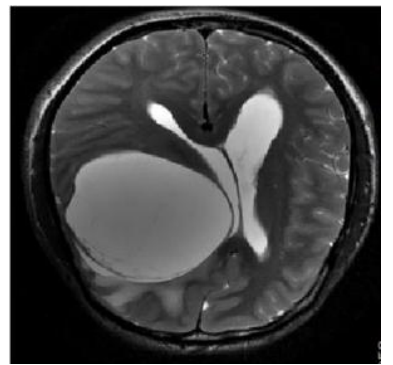

Image (1)

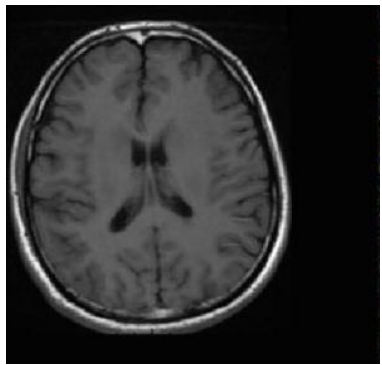

Image (2)

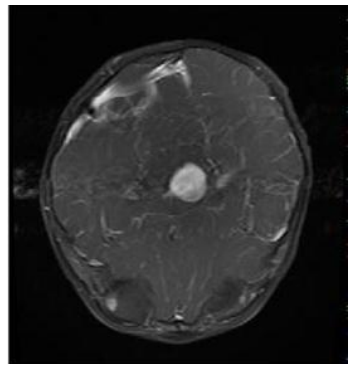

Image (3)

Figure 1 Brain tumor input images

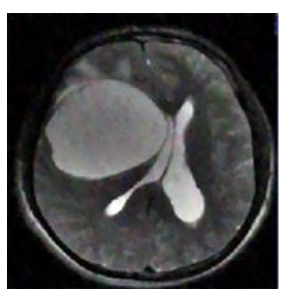

1(a) Original Image

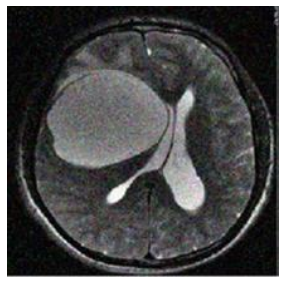

1(b) Noisy image

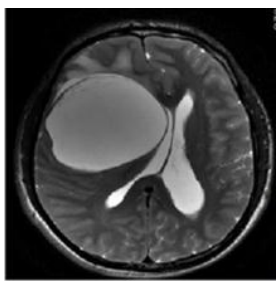

1(c) denoised image

(Conventional method)

Figure 2 Processing through proposed method of image 1 of figure 1

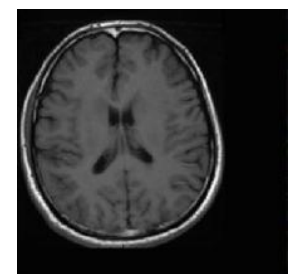

2(a) Original Image

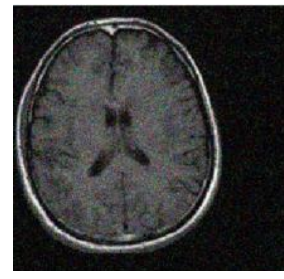

2(b) Noisy image

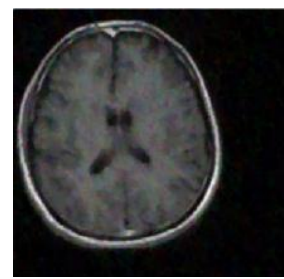

2(c) Denoised image (Conventional method)

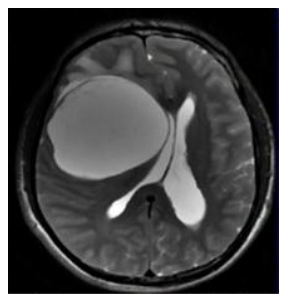

1 (d) denoised image (Proposed method)

Figure 3 Processing through proposed method of image 2 of figure 1

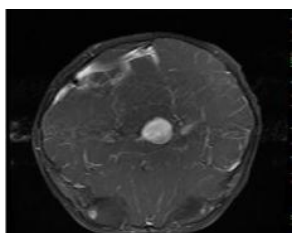

3(a) Original Image

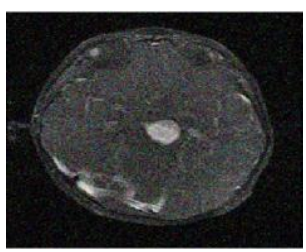

3(b) Noisy image

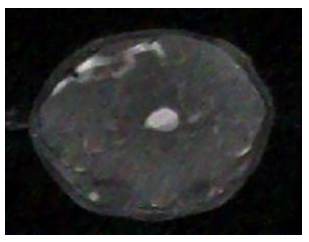

3(c) Denoised image (Conventional method)

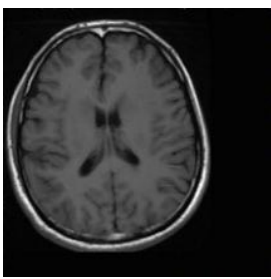

2(d) Denoised image (Proposed method)

Figure 4 Processing through proposed method of image 3 of figure 1

3.2Steps to be performed for image denoising

- Apply the kth disintegrations on discrete wavelet transform (DWT) for an image $\mathrm{n}$ to transform into noisy wavelet coefficients $\mathrm{F}$.

- Evaluate the noise variance $\sigma^{2}$ by equation 4 .

- Assess $S_{\text {new }}$ from equation 8 at every high sub band, and put on soft-threshold to the wavelet coefficients.
- Apply inverse discrete wavelet transform to obtain reconstructed image $\hat{f}$.

\section{Results and discussion}

We have run a sequence of experiments using MATLAB R2013a. By taking brain tumor images as an input. Image 1 having a tumor on left side in the brain. Tumors present on both sides of brain in image 
2 and right side of brain in image 3. Gaussian noise at different level of variance 5, 10, 15 and 20 has been added to all three images. It generates the noisy images displayed in Figures $1(b), 2(b)$ and $3(b)$. The first image i.e. (a) presents the original one, and the second image i.e. (b) presents the noisy image with Gaussian noise level 20 as shown in Figures 2-4. The third ones (c) are the denoised images applying the conventional method [5] of denoising and the fourth image in a row i.e. (d) are the denoised images using our proposed method. It is manifest from these figures that the denoise images using our proposed method have superior visual quality as compared to the available conventional methods.
The quality of test images is measured in terms of PSNR. The experimental results of our proposed method are depicted in Table 1. These PSNRs specify that our proposed method is better to the existing method. As we increase the noise level, denoising effect of conventional method decreases, but the proposed method contributes better execution with high noise. From the results obtained in Table 1 and Figures 2-5 verify that, our method achieves better edge effects and eliminate noise well as compared with available methods.

Table 1 Results in terms of PSNR (in $\mathrm{db}$ ) for brain tumor images

\begin{tabular}{lccc}
\hline \multirow{2}{*}{ Image name } & Noise level & Methods \\
\cline { 2 - 4 } & 5 & 28.63 & Proposed \\
\hline & 10 & 26.70 & 35.56 \\
& 15 & 25.45 & 32.48 \\
$(1)$ & 20 & 22.01 & 30.23 \\
& & & 30.06 \\
& 5 & 26.60 & 39.65 \\
& 10 & 25.94 & 35.13 \\
& 15 & 23.10 & 32.89 \\
& 20 & 21.45 & 31.18 \\
$(3)$ & & & 36.65 \\
& 5 & 28.15 & 33.23 \\
& 10 & 23.14 & 32.09 \\
\end{tabular}

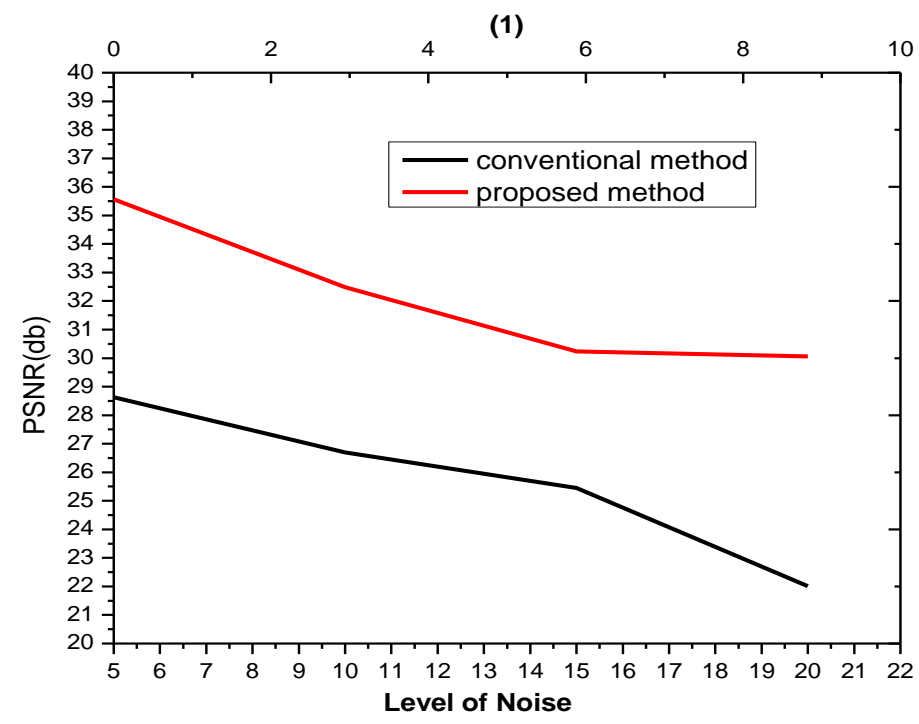



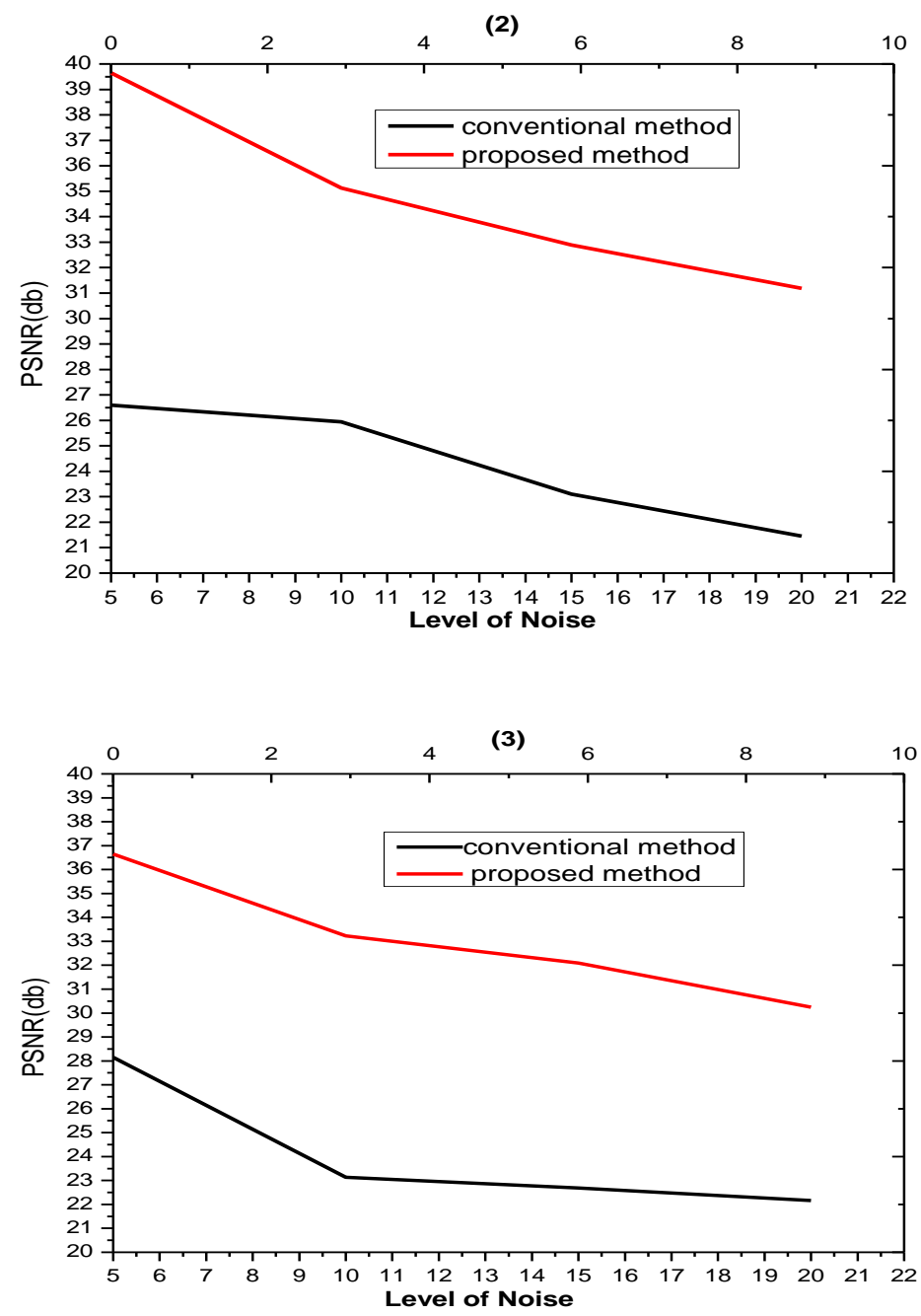

Figure 5 PSNR vs. noise levels of conventional and proposed methods with images (1), (2) and (3)

\section{Conclusion}

In this paper a new thresholding technique that decreases gaussian noise significantly from a noisy image has been introduced. In addition to that, it improves considerably the visual quality of the noisy image. In future to achieve higher PSNR and more clarity in images other techniques may be applied as suggested in [18]. Our approach can be improved in the direction of hybrid approaches for better denoising results [19].

\section{Acknowledgment}

The authors would like to acknowledge their friends and colleagues for their constant help and support during the study and to acquire the outcomes.

\section{Conflicts of interest}

The authors have no conflicts of interest to declare.

\section{References}

[1] Bruni V, Vitulano D. Combined image compression and denoising using wavelets. Signal Processing: Image Communication. 2007; 22(1):86-101.

[2] Bui TD, Chen G. Translation-invariant denoising using multiwavelets. IEEE Transactions on Signal Processing. 1998; 46(12):3414-20.

[3] Chen GY, Bui TD, Krzyzak A. Image denoising using neighbouring wavelet coefficients. Integrated Computer-Aided Engineering. 2005; 12(1):99-107.

[4] Bhandari AK, Kumar D, Kumar A, Singh GK. Optimal sub-band adaptive thresholding based edge preserved satellite image denoising using adaptive differential evolution algorithm. Neurocomputing. 2016; 174:698-721. 
[5] Elyasi I, Zarmehi S. Elimination noise by adaptive wavelet threshold. World Academy of Science, Engineering and Technology. 2009; 3(8):1541-5.

[6] Donoho DL. De-noising by soft-thresholding. IEEE Transactions on Information Theory. 1995; 41(3):61327.

[7] Donoho DL, Johnstone IM. Adapting to unknown smoothness via wavelet shrinkage. Journal of the American Statistical Association. 1995; 90(432):120024.

[8] Cai TT, Silverman BW. Incorporating information on neighbouring coefficients into wavelet estimation. Sankhyā: The Indian Journal of Statistics, Series B. 2001; 63(2):127-48.

[9] Zhang Y, Tian X, Ren P. An adaptive bilateral filter based framework for image denoising. Neurocomputing. 2014; 140:299-316.

[10] Dabov K, Foi A, Katkovnik V, Egiazarian K. Image denoising by sparse 3-D transform-domain collaborative filtering. IEEE Transactions on Image Processing. 2007; 16(8):2080-95.

[11] He K, Sun J, Tang X. Guided image filtering. IEEE Transactions on Pattern Analysis and Machine Intelligence. 2013; 35(6):1397-409.

[12] Dong W, Ding H. Full frequency de-noising method based on wavelet decomposition and noise-type detection. Neurocomputing. 2016; 214:902-9.

[13] Liu X, Wan H, Shang Z, Shi L. Automatic extracellular spike denoising using wavelet neighbor coefficients and level dependency. Neurocomputing. 2015; 149:1407-14.

[14] Ordentlich E, Seroussi G, Verdu S, Weinberger M, Weissman T. A discrete universal denoiser and its application to binary images. In international conference on image processing 2003 (pp. 117-20). IEEE.

[15] Nasri M, Nezamabadi-pour H. Image denoising in the wavelet domain using a new adaptive thresholding function. Neurocomputing. 2009; 72(4-6):1012-25.
[16] Srisailam C, Sharma P, Suhane S. Color image denoising using wavelet soft thresholding. International Journal of Emerging Technology and Advanced Engineering. 2014; 4(7):475-8.

[17] Hongqiao L, Shengqian W. A new image denoising method using wavelet transform. In international forum on information technology and applications 2009 (pp. 111-4). IEEE.

[18] Gupta D, Ahmad M. An efficient method to get improved peak signal to noise ratio (PSNR), using support vector machine. International Journal of Emerging Technology and Advanced Engineering. 2017; 7(9): 49-53.

[19] Gupta D, Ahmad M. A hybrid technique based on fuzzy methods and support vector machine for prediction of brain tumor. International Journal on Computer Science and Engineering.2017; 9(8): 51721.

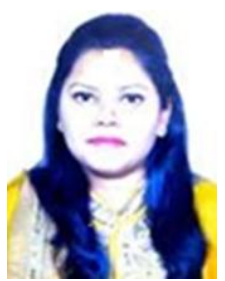

Deepti Gupta has completed M.Sc. (Mathematics) from DBRAU Agra, Currently, she is pursuing Ph.D. in the field of Applications of support vector machine and wavelets in image processing from Jamia Millia Islamia, New Delhi, India.

Email:dg.gupta399@gmail.com

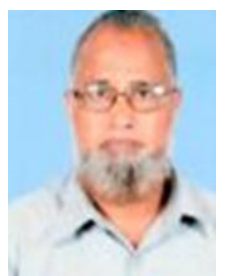

Prof Musheer Ahmad is working as Head of the Department of Applied Sciences \& Humanities at Jamia Millia Islamia, New Delhi, India. He is Ph.D. and M.phil from Department of Mathematics, Aligarh Muslim University, India. His current research interests include Fuzzy algebra, Graph theory, Image Processing, Soft computing and Information Security. 